\title{
Die Grenzen des Systems und die Rhetorik der Systemtheorie
}

Wer die kulturwissenschaftliche Theoriebildung der letzten Jahrzehnte Revue passieren läßt, wird bald auf gemeinsame Merkmale stoßen. Solche Gemeinsamkeiten sind leichter am Stil als an der jeweiligen argumentativen Durchführung zu erkennen. Sie bestehen darin, innerhalb hergebrachter Begriffsoppositionen der bis dahin schwächeren Seite den Vorzug zu geben. Positiv akzentuiert werden nun die Differenz, das Uneinheitliche, Unbeständige, Nichtzusammenhängende, Widerstreitende, Heterogene. Damit geht der Abbau einer jahrhundertealten Raummetaphorik einher, die bestimmte Orte privilegiert hatte: Mitte, Höhe (als Blickhöhe und als Machtposition) und Tiefe (als Tiefe des Grundes). Diese zentristischvertikale Topologie schwächt sich $a b$ und weicht einer flächenhaften Anlage des epistemischen Feldes, verbunden mit einer besonderen Hochschätzung der Peripherie. Den aktuellen Kulturtheoretikern ist es um das Abschreiten von Rändern zu tun - um den Aufenthalt in jener gefährlichen Zone, in der Ordnung in Unordnung, Vertrautheit in Fremde umschlägt. Und zwar nicht deshalb, um auf diesem Weg wieder überschaubare Terrains einzugrenzen, sondern um die Auflösung der klassischen Ökonomie der Grenze und ihrer Innen/AußenScheidung voranzutreiben.

Durch die Umstellung vom Primat der Einheit auf den Primat der Unterscheidung, die Luhmann in den achtziger Jahren vollzog, durch Schlüsselkategorien wie Kontingenz und Unwahrscheinlichkeit ist die Systemtheorie in diesen allgemeinen Trend involviert. Das führt zu ihrer Annäherung an ganz anders geartete Theoriemilieus - etwa die Dekonstruktion. Auch die Systemtheorie wird von anti-ontologischen und anti-metaphysischen Impulsen getragen, und sie teilt mit neueren Kulturtheorien die Einsicht in den Konstruktionscharakter dessen, was gemeinhin ,Realität' genannt wird. Das trägt ihr und damit der Soziologie überhaupt das Problem der Referenz ein, an dem die Kulturwissenschaft als Textwissenschaft schon seit längerem laboriert. Nur daß sie dieses Problem nicht semiologisch angeht, sondern als Problem der Beobachtung, genauer gesagt der Beobachtung zweiten Grades, das heißt der Beobachtung eines ersten (naiven) Beobachters.

Dennoch bewahrt Luhmanns Theorie einen eigenen, man könnte fast sagen: scholastischen, Zug, der sie in klaren Kontrast zu den genannten Entwicklungen stellt. Und das hängt mit ihrem auffälligen Reinheitsbegehren bei der Beschreibung von Systemoperationen zusammen. So sehr Luhmann Systeme von ihrer Grenze her denkt - nämlich der System/Umwelt-Differenz -, so wenig verbinden sich damit für ihn jene tiefen und infektiösen 
Zweideutigkeiten des Randes, der Schwelle, des Ausgeschlossenen und Aufgeschobenen dessen gedankliche und stilistische Möglichkeiten zumal Derridas Texte entfalten. Fü Luhmann hält die Systemgrenze die internen Operationen des Systems von Fremdeinwirkungen rein. Deshalb kann er, selbst bei mehrfach konditionierten Ereignissen, zwischen den Referenzen auf die Teilsysteme (Recht, Wirtschaft, Religion, Wissenschaft etc.) und ihre jeweiligen Codes säuberlich unterscheiden. In funktionaler statt hierarchischer Untergliederung tauchen so schemenhafte Züge der alten ordo mundi wieder auf - eine geradezu prästabilierte Ordnung der Funktionswelten, scheinbar frei von all jenen unkontrollierbaren Mischformen an den Rändern, derer sich die poststrukturalistische Textlehre nicht erwehren kann.

Um diese ,Grenzhygiene ' bei Luhmann werden die folgenden Überlegungen kreisen. Sie sollen den Nachweis erbringen, daß auch Luhmanns Theoriestil der verunreinigenden Wirkung von Grenzziehungen nicht entrinnt. Das betrifft den System/Umwelt-Bezug, aber es gilt ebenso in der Zeitperspektive: nämlich mit Blick auf die Tatsache der ,unreinen Geburt des Systems. Anders formuliert, mit Blick auf die Diskrepanz und damit Unreinheit der Kategorien, mit denen allein der Anfang eines Systems zu beschreiben ist. Luhmanns Systemfunktionalismus wird hier sozusagen von rhetorischen Komplikationen unterwandert die auf die textuelle Verfaßtheit seiner eigenen Theorie zurückdeuten. Texte im vollen Wortsinn sind plurale, a-systemische Gebilde. Sie verfügen über keinen eindeutigen Systembezug; ihre Polyvalenz wird nicht durch einen isolierbaren binären Code und dessen Unterprogramme gesteuert. Textuelle Strukturen im weitesten Sinn dieses Begriffs, wie ihn die neueren Kulturwissenschaften verwenden, sind deshalb nur unvollständig in systemrationale Vorgänge übersetzbar.' An ihrem entscheidenden Punkt, das soll gezeigt werden bricht die Logik der Systemtheorie auf und wird durchkreuzt von einer Logik der Kultur.

Luhmann zufolge sind soziale Systeme durch die Verschränkung zweier gegensätzlicher Eigenschaften bestimmt. Sie sind sowohl offen als auch geschlossen - offen, insofern sie Umweltreizen ausgesetzt sind; geschlossen durch ihre rekursive Struktur, die sie darauf beschränkt, Sequenzen von systeminternen Operationen zu bilden. Diese widersprüchlichen Bestimmungen lassen sich nicht durch Gradation, durch ein Mehr oder Weniger an Offenheit schlichten. Das hängt mit dem Konzept der Autopoiesis zusammen, das Luhmann in die Gesellschaftstheorie eingeführt hat.

Autopoietische Systeme sind nämlich nicht offen in dem Sinn, daß sie einen Austausch mit ihrer Umwelt unterhalten. Zwar bleiben sie abhängig von Umweltbedingungen, die ihre Existenz gewährleisten, aber das betrifft nicht die operative Ebene ihrer Selbstreproduktion. Anders als nach dem black-box-Modell empfangen sie keine Inputs und geben keine Outputs zurïck. Ihr Systemcharakter schließt aus, daß sie von ihrer Umwelt direkt affiziert werden können. Insofern alle Operationen eines Systems selbstreferentiell allein an vorherige Operationen desselben Systems anschließen, finden sie notwendig innerhalb undurchlässiger Systemgrenzen statt.

1 Vgl. die Überlegungen, die Hayles mit Blick auf Maturana anstellt: N. Katherine Hayles, „Making the Cut: The Interplay of Narrative and System, or What Systems Theory Can't See“, in Cultural Critique, $30,1995,71-100$
Luhmann hat in seiner mehr als dreißigjährigen soziologischen Arbeit erst relativ von den Sozialen Systemen (1984) an, zu einer derart rigiden Fassung des Problem Systemgrenze gefunden. Man kann diese Entwicklung systematisch nachvollziehen; sie sich aber auch an stilistischen Merkmalen ablesen, nämlich an den Grenzmetaphern, die seine jeweilige Darstellung prägen. Luhmanns frühe verwaltungstheoretische Schrift Funktionen und Folgen formaler Organisation von 1964 räumt zwar dem bürokratischen Apparat eine eigenständige, gegen systemfremde Vorgaben weitgehend indifferente Rationalität ein, sieht aber noch Vermittlerrollen zwischen dem Verwaltungsbetrieb und seiner Außenwelt vor. ${ }^{2}$ Spätestens seit den Sozialen Systemen legt Luhmann auf die Feststellung Wert, daß die Grenze keinen dritten Bereich zwischen System und Umwelt bilde, sondern vom System aus modelliert werde. ${ }^{3}$ Dennoch ist er in seiner Metaphorik noch nicht entschieden; sie spielt die ganze Bandbreite zwischen Öffnung und Schließung durch. „Es handelt sich [...] vom System aus gesehen um ,self-generated boundaries', um Membranen, Häute, Mauern und Tore, Grenzposten, Kontaktstellen. .c4 $^{4}$

Solche Vergleiche suggerieren noch einen, wenn auch quantitativ und strukturell eingeschränkten, System-Umwelt-Verkehr. In dieser Phase kann Luhmann Systemgrenzen als ,hochselektive Kontaktverengungen" bezeichnen, ohne die Tatsache des Kontakts selbst zu bestreiten. ${ }^{5}$ Er kann die ,allgemeine Regel“ aufstellen, „daß Systeme nicht nur aus Punktfür-Punkt-Beziehungen zur Umwelt bestehen ${ }^{\star 6}$ und damit einen Anteil von unmittelbaren System-Umwelt-Bezügen einräumen. Er kann schließlich sogar von „Importen aus der

2 Niklas Luhmann, Funktionen und Folgen formaler Organisation, Berlin 1964. Darin bes. das Kapitel "Grenzstellen“, 220ff. Luhmann analysiert die Rollenbedingungen für „Mitglieder in Grenzstellen“ von Verwaltungsapparaten und schreibt: „Sie empfangen die Verhaltenserwartungen, welche die Umwelt an das System adressiert, sozusagen im Rohzustand und unverfälscht, bevor also die Information durch mehrere Hände gelaufen ist und sich den im System herrschenden Annahmen über die Umwelt angeglichen hat [...].“ (221) Grenzstellen leisten Übersetzungsarbeit. Weil nämlich das System, um zu bestehen, seine Grenzen invariant halten muß, können „sich Umwelterwartungen nicht natürlich und unverändert in das System hinein fortsetzen [...]. Ein Bruch an den Grenzen ist unvermeidlich, darf aber gleichwohl das System nicht gefährden." (223)

Die Erfüllung der Grenzstellenfunktion erfordert eine notwendigerweise grenzüberschreitende Aktivität. Warnung des Systems. Diese Seite ihres Auftrages zwingt sie Verantwortung für neue Informationen zu übernehmen, Initiative zu ergreifen, Rollenverpflichtungen über die Grenze hinweg einzugehen [...]““ (224) - „Die Grenzstellen interpretieren die Umwelt für das System. Sie müssen Umweltinformationen sichten und sieben und sie in eine Sprache bringen, die im System verstanden und akzeptiert wird." (ebd)

Es handelt sich bei all dem nicht um punktuelle, sondern komplexe Funktionen, und so haben Grenzstellen eine Neigung dazu, sich zu einem ,Zwischensystem“ (226) mit doppelseitigen Loyalitäten zu entwikkeln: „Aus einer Trennlinie wird eine verbreiterte Zwischenzone relativ freien, nicht voll verantwortlichen, aber auch nicht voll verbindlichen Handelns, die von beiden Seiten in ihrer besonderen Eigenart respektiert wird." (229)

3 Niklas Luhmann, Soziale Systeme, Grundriß einer allgemeinen Theorie, Frankfurt am Main 1988, 53.

4 Ebd., 53f. „In entwicklungsdynamischer Perspektive gesehen“, schreibt Luhmann weiter, ,sind Grenzen steigerbare Leistungen. Diesen Aspekt haben wir mit dem Begriff der Ausdifferenzierung von Systemen bezeichnet. Die Grenzbildung unterbricht das Kontinuieren von Prozessen, die das System mit seiner bezect ter Dinden. Die Steigerung der Grenzleistung besteht in der Vermehrung der Hinsichten, in dener Umwelt verbinden. Die Steigerung der Grenzleistumg besteht in der Vert dies geschieht." (54)

5 Niklas Luhmann, Funktion der Religion, 4. Auflage, Frankfurt am Main 1996 [zuerst 1982], 14.

6 Ebd., 23. 
Umwelt, etwa Wissen" im Stil einer eigentlich schon überwundenen informationellen Mechanik sprechen.

Je mehr Ordnungskompetenz indessen an das System übergeht, desto weniger Eigenstruktur können von außen kommende Anstöße mitbringen beziehungsweise bei ihrem Eintritt in die Systemsphäre bewahren. Und folglich verlieren solche topographischen Modelle überhaupt ihre Gültigkeit. Die Grenze hört auf, als Schwelle oder Übergangszone beschreibbar zu sein, sie hat keine „Tore“ und keine „Kontaktstellen“ mehr. Das System kann nur noch per Resonanz, durch Aufmerksamkeit auf seine Eigenfrequenzen, auf Gegebenheiten der Umwelt reagieren. ${ }^{8}$

Das Konzept der Selbstorganisation stuft die Umwelt zur „Quelle eines unspezifischen (sinnlosen), Rauschens" " herab, das nach dem kybernetischen Motto order from noise allein durch die Selektions- und Kombinationsleistung des Systems in sinnvolle Information transformiert werden kann. ${ }^{9}$ Doch selbst bei einer solchen Datenselektion bleibt Luhmann nicht stehen. Sie wird durch den Begriff der Autopoiesis in seinem vollen Verständnis noch überboten.

Autopoietische Systeme", heißt es in Luhmanns letztem Werk Gesellschaft der Gesellschaft, ,sind Systeme, die nicht nur ihre Strukturen, sondern auch die Elemente, aus denen sie bestehen, im Netzwerk eben dieser Elemente selbst erzeugen." $(G d G, 65)$ Auch wenn diese Elemente, wie bei psychischen und sozialen Systemen, in kognitiven und kommunikativen Akten bestehen, stellen sie keinerlei Objektbezug her. Luhmann weist ,auf diesen schwierigen Gedanken, der der gesamten erkenntnistheoretischen Tradition widerspricht $(G d G, 92)$, mit besonderem Nachdruck hin. „Beobachtungen können nur auf Beobachtungen einwirken, [...] können, mit anderen Worten, nur Informationen verarbeiten; aber nich Dinge der Umwelt berühren [...]. Auch für beobachtende Systeme gibt es auf der Ebene ihres Operierens keinen Umweltkontakt " (ebd.) Wissen, Information, Beobachtung, Zeichen sind nun, zumindest dem Anspruch der Theorie nach, intransitive Größen geworden.

Man sollte sich zunächst die Leistungen des Modells der Autopoiesis vergegenwärtigen, bevor man die Analyse der ihm inhärenten Schwierigkeiten weitertreibt.|Systeme, das ist die Ausgangsüberlegung, können ihre Funktion dann und nur dann erfüllen, wenn sie äußere Komplexität reduzieren und innere, das heißt strukturelle Komplexität aufbauen. ${ }^{10}$ Ein System ohne hinreichende Abschirmung von den unendlich vielfältigen Vorgängen in seine Umgebung würde aufhören, sich zu reproduzieren; es würde an einer nicht zu verarbeitenden Übermenge an Umwelteinflüssen zugrundegehen Es kann sich nur um den Preis der Grenzziehung und Ausbildung eigener konsistenter Strukturen, das heißt durch Ruickbezüglichkeit auf sich selbst, operationsfähig machen. Es schaltet von Fremd- auf Selbstanpas-

7 Ebd., 31.

Vgl. Niklas Luhmann, Ökologische Kommunikation. Kann die moderne Gesellschaft sich auf ökologische Gefährdungen einstellen? Opladen 1988, 41.

9 Niklas Luhmann, Die Gesellschaft der Gesellschaft, 2 Teilbände, Frankfurt am Main 1997, 65 (im folgenden unter der Sigle $G d G$ mit Angabe der Seitenzahl im laufenden Text zitiert).

10 Vgl. Niklas Luhmann, Soziale Systeme (Anm. 3), 45ff. sung um" und wird gerade durch Abweichung von Umweltvorgaben zu komplexen kognitiven Leistungen befähigt. Weil „kein System seine Umwelt beobachten (oder allgemeiner: Kognition entwickeln) könnte, wenn es jedes Ereignis der Umwelt durch einen Eigenzustand parieren müßte ${ }^{612}$, läßt sich im Umkehrschluß allein durch operative Geschlossenhei Offenheit gegenüber der Außenwelt erreichen. Die beiden Attribute Geschlossenheit und Offenheit widersprechen sich also nicht, sie bedingen einander. „Das System gewinnt seine Freiheit und seine Autonomie der Selbstregulierung durch Indifferenz gegenüber seine Umwelt. Deshalb kann man die Ausdifferenzierung eines Systems auch beschreiben als Steigerung der Sensibilität für Bestimmtes (intern Anschlußfähiges) und Steigerung der Insensibilität für alles übrige - also Steigerung von Abhängigkeit und Unabhängigkeit zugleich. ${ }^{\text {"13 }}$

In diesen Zusammenhang gehört noch eine weitere Basisannahme der neueren Systemtheorie. Sie versteht Systeme bekanntlich nicht mehr als mit sich übereinstimmende Ganzheiten, sondern differentiell, eben in der Unterschiedenheit von ihrer jeweiligen Umwelt. „Systeme“, so Luhmann, ,sind nicht nur gelegentlichyind nicht nur adaptiv, sie sind strukturell an ihrer Umwelt orientiert und können ohne Unwelt nicht bestehen. Sie konstituieren und sie erhalten sich durch Erzeugung und Erhaltung einer Differenz zur Umwelt, und sie benutzen ihre Grenzen zur Regulierung dieser Differenz. Ohne Differenz zur Umwelt gäbe es nicht einmal Selbstreferenz, denn Differenz ist Funktionsprämisse selbstreferentieller Operationen. “14 Dieses Axiom einer konstitutiven Bezogenheit des Systems auf das, was es nicht ist, erübrigt, so glaubt jedenfalls Luhmann mit Zuversicht, erkenntnistheoretische Probleme, an denen sich die Philosophie bisher abgearbeitet hat: der Konstruktivismus als epistemologisches Seitenstück der Systemtheorie mache ,die alten Einwände gegen einen vermeintlich realitätslosen Idealismus" hinfällig $(G d G, 98)$.

Autopoiesis entfaltet sich als Entfaltung der System/Umwelt-Differenz, oder, anders formuliert, der Differenz zwischen Selbstreferenz und Fremdreferenz; sie spielt also beständig mit der eigenen Grenze. Und dennoch bleibt das Problem, wie die Umwelt ,ins System oder umgekehrt das System an seine Umwelt, heran' kommt, ungelöst. Es taucht hinter jeder schlichtenden Formulierung, die Luhmann findet, von neuem auf. Das hat einen einfachen systematischen Grund. Denn die Differenz zwischen Selbstreferenz und Fremdreferenz ist ihrerseits nur handhabbar, insoweit sie innerhalb des Systems, innerhalb seiner Grenzziehungen und damit in gewisser Weise diesseits seiner Differentialität gehandhabt wird; sie besteht in einer Unterscheidung, die das System trifft und sozusagen allein mit sich ausmacht. „Alle Umweltbeobachtung muß im System selbst als interne Aktivität mit Hilfe eigener Unterscheidungen (für die es in der Umwelt keine Entsprechung gibt) durchgeführt werden." $(G d G, 92)$ Das System beziehungsweise der Beobachter im System schreibt Fremdreferenz $z u$, und zwar durch wahlweise erfolgende Externalisierungen. ${ }^{15}$ Die von de klassischen Metaphysik her naheliegende Frage nach der Garantie derartiger Zuschreibungen durch eine grenzübergreifende Instanz ist im Vokabular der Systemtheorie weder zu beantworten, noch kann sie überhaupt sinnvoll gestellt werden.

1 Ebd., 56.

12 Niklas Luhmann, „Wie ist Bewußtsein an Kommunikation beteiligt?", in Hans-Ulrich Gumbrecht und Karl Ludwig Pfeiffer (Hg.), Materialität der Kommunikation, Frankfurt am Main 1995, 894.

13 Niklas Luhmann, Soziale Systeme (Anm. 3), 250.

13 Niklas Luhm. 35 .

15 Vgl. Niklas Luhmann, Die Wissenschaft der Gesellschaft, Frankfurt am Main ${ }^{2} 1994,291$ und Anm. 33. 
Systemoperationen dazu veranlassen soll, einen solchen inneren Reibungswiderstand $z u$ erzeugen.

Ein Beispiel soll verdeutlichen, mit welchen elementaren gedanklichen und infolgedessen auch terminologischen Schwierigkeiten das Modell der Systemgrenze zu kämpfen hat. Ein wichtiger Indikator für die Funktionsweise dieser Grenze ist der Begriff der Irritation. "Autopoietische Systeme“, schreibt Luhmann in Die Gesellschaft der Gesellschaft, "reagieren unmittelbar auf negative bzw. nicht typisierbare Reize." $(G d G, 118)$ Sie scheinen also überraschbar zu sein. Einerseits. Andererseits aber funktionieren sie autopoietisch, und das macht sie nach außen hin indifferent. „Auch in ihrer Irritierbarkeit", heißt es gleich darauf, ,sind die Systeme, und zwar sowohl die Bewußtseinssysteme als auch das Kommunikationssystem Gesellschaft, völlig autonom. Irritationen ergeben sich aus einem internen Vergleich von (zunächst unspezifizierten) Ereignissen mit eigenen Möglichkeiten, vor allem mit etablierten Strukturen, mit Erwartungen. Somit gibt es in der Umwelt des Systems keine Irritation, und es gibt auch keinen Transfer von Irritation aus der Umwelt in das System. Es handelt sich immer um ein systemeigenes Konstrukt, immer um Selbstirritation - freilich aus Anlaß von Umwelteinwirkungen." (ebd.)

Irritation findet im System statt. Aber das Problem der Kausalität ist nur verschoben: „freilich aus Anlaß von Unwelteinwirkungen“. Nur: wie wirkt Umwelt ein? ,Beeindruckt" sie das System? Das würde heißen, es in seiner Autonomie zu beeinträchtigen. Determiniert sie die Irritation? Das würde „Selbstirritation“ zu einer terminologischen Floskel herabstufen und der Forderung nach „Brechung der externen Determination“ durch „operative Schließung" des Systems widersprechen. ${ }^{16}$ Aber ohne jede Beeinträchtigung hätte das System überhaupt keinen Anlaß, sich über sich selbst zu irritieren. ${ }^{17}$ Daran ändert die salomonische Formel nichts, die der Konstruktivist Luhmann bezeichnenderweise von der Dekonstruktion übernimmt. Es ist immer das gleiche Zitat, und gewissenhafte LuhmannLeser sind ihm schon häufig begegnet. Gemeint ist die Formel von der ,resistance of language to language", mit der Wlad Godzich Paul de Mans , Resistance to Theory" kommentiert (zuletzt in: $G d G, 95$ ).

Oder, systemtheoretisch reformuliert und nicht auf Sprache, sondern „Beobachtungen“ bezogen: „Ihr Realitätswert liegt [...] nicht, wie die gesamte an Erkenntnis interessierte Tradition annahm, in der Realität ihrer Gegenstände, die entweder wahr oder unwahr, entweder zutreffend oder unzutreffend beobachtet und beschrieben werden. Sie liegt vielmehr ausschließlich in der Realität der Beobachtungsoperationen selbst, das heißt im Austesten eines Widerstandes, der nicht in einer gegenständlichen Außenwelt liegt, sondern ausschließlich in der rekursiven Vernetzung der Systemoperationen selbst." $(G d G, 538)$ Und wieder steht man am Ausgangspunkt der immergleichen Spirale und fragt sich, was die

16 Niklas Luhmann, Die Realität der Massenmedien, Opladen ${ }^{2} 1996,56 f$.

17 Das Gleiche läßt sich in bezug auf den Begriff der Resonanz zeigen. Auch hier muß Luhmann das Moment der Beeinträchtigung, der Passivität des Systems exkamotieren, wenn ,Resonanz ${ }^{6}$ mit ,Autonomie' kompatibel sein soll. Das führt zu sprachlichen Kompromißlösungen wie bei der Forme von einer ,selbstbestimmten Resonanzfähigkeit des Wirtschaftssystems“, die Luhmann in der Ökologischen Kommunikation verwendet (ebd. (Anm. 8), 122).

Selbst einfache Rückkopplungen werden auf diesem Weg kategorial unbeschreibbar. Beispiel HausSelbst einfache Rickkopplungen worden auf diesem Weg kategoral unbeschreibbar. Beispiel Haustechnik: „In der Außenwelt gibt es eben keine Temperaturen - womit nicht geleugnet sein soll, daß man nicht anspringt." (Niklas Luhmann, Die Wirtschaft der Gesellschaft, Frankfurt am Main ${ }^{2} 1996,335$ )
In anderen Wendungen macht Luhmann die systematische Umweltblindheit der ausdifferenzierten Funktionssysteme ganz explizit. „Die Systeme operieren mithin unter der Illusion eines Umweltkontaktes“, heißt es dann $(G d G, 93)$, „,im imaginären Raum“ der Referenzen $(G d G, 98)$. „Die Referenz auf ,die Umwelt" trägt nichts zu den Systemoperationen bei. ,Die Umwelt" gibt keine Information. Sie ist nur ein Leerkorrelat für Selbstreferenz " (GdG, 609) Noch ein letztes Zitat hierzu, um die Sammlung zu vervollständigen: „Die Außenwelt ist, wie sie ist: stur, möglichkeitslos und unbekannt." ${ }^{\text {"18 }}$

Es liegt durchaus in Luhmanns Vermögen, dieses blinde und an alte anthropozentrische Modelle erinnernde Alleinsein in der Welt mit einer gewissen tragischen Grandiosität zu vermerken: „Und wenn die Gesellschaft aus der Gesamtheit aller Kommunikationen besteht, ist die übrige Welt zur Sprachlosigkeit verurteilt. Sie zieht sich ins Schweigen zurück. Ja nicht einmal das ist ein angemessener Begriff, da Schweigen nur kann, wer kommunizieren könnte." $(G d G, 158)$

Aber er hat auch ein anderes und öfter verwendetes Stilregister, das einer sich autistisch gebenden Unterkühlung: „Irritation ist ein jeweils systemeigener Zustand ohne Entsprechung in der Umwelt des Systems. Wenn man an einem System eine Irritation beobachtet, kann man daraus nicht schließen, daß auch die Umwelt entsprechend irritiert sei; ja nicht einmal, daß der Umweltzustand, der die Irritation auslöst, für die Umwelt (für wen denn?) ein Problem sei. A ,pollution' is a creation of human judgment. Das Ozonloch, das gesunkene U-Boot mit Nuklearantrieb, die ,sterbenden ' Wälder sind nicht über sich selbst irritiert. Die Umwelt ist, wie sie ist." $(G d G, 792)^{19}$

5.

Formulierungen dieser Art bieten ausreichenden Anlaß zu einer Art Ideologiekritik de Systemtheorie. Zu einer Zeit, in der Umweltprobleme längst zu globalen Überlebensfragen geworden sind, verleiht Luhmann der ökologischen Betriebsblindheit der modernen Gesellschaft sozusagen eine methodologische Weihe. ${ }^{20}$ Seine Antworten auf solche Probleme sind

18 Niklas Luhmann, Wirtschaft der Gesellschaft, 334.

19 Anderswo ist - Umwelttatsachen gibt es für Luhmann nicht - von „sagenhafte[n] Ozonlöcher[n]“ die Rede (Wissenschaft der Gesellschaft (Anm. 15), 654). Allerdings heißt es unmittelbar im Anschluß an die zitierte Passage in souveräner Inkonsequenz: „Was immer man von dieser Theoriekonstellation halten mag: zu beobachten ist, daß die Irritationsanläße [sic] aus der Umwelt des Gesellschaftssystems in den letzten Jahrzehnten dramatisch zunehmen - und zwar auch und gerade auf den Bildschirmen der Gesellschaft selbst.“ $(G d G, 795)$ Zur Metapher der „Bildschirme“ der Gesellschaft wäre anzumerken, daß sie wiederum mit dem Begriff der Systemautonomie kollidiert. Was nämlich diese Autonomie betrifft, ,gibt es keine Halbheiten oder Abstufungen, keine Relativierungen, kein mehr oder wenigerDenn entweder produziert das System seine Elemente selbst oder nicht. Wenn es, wie ein Computer, Elemente oder Stuk (lemente kein autopoietisches System. " (Niklas Luhmann, Die Kunst der Gesellschaft, Frankfurt am Main 1995, 254) Woher sollten „Irritationsanlässe“ auf die „Bildschirme der Gesellschaft“ gelangen, wenn man nur von der Eigenfrequenz der Monitore, nicht aber von einer externen Datenquelle ausgehen darf?

20 Vgl. Hans Peter Dreitzel und Horst Stenger (Hg.), Ungewollte Selbstzerstörung. Reflexionen über den Umgang mit katastrophalen Entwicklungen, Frankfurt am Main, New York 1990. Dort die Einleitung, bes. $16 \mathrm{ff}$. 
im wesentlichen bürokratischer Natur, nämlich Unzuständigkeitserklärungen; sie verraten, daß seine universale Gesellschaftstheorie in gewissen Zïgen noch immer die niedersächsische Verwaltungswissenschaft ist, als die sie begann.

Doch reichen derartige Vorhaltungen nicht aus, um Breschen in die Triftigkeit und innere Konsequenz des systemtheoretischen Gedankengebäudes zu schlagen. Im übrigen teilt die Systemtheorie gerade ihr grundsätzliches epistemologisches Dilemma mit jenen aus dem Strukturalismus und damit aus einem ihr ganz fernen Milieu hervorgegangenen Theorievarianten, die an der unumgehbaren Selbstbezüglichkeit von Zeichenprozessen herumlaborieren. Und den zirkulären Argumentationen etwa Paul de Mans, die jeden Transgressionsversuch des Sprechens nur immer wieder im Gefängnis der Sprachlichkeit einmünden lassen, ist die Systemtheorie durch ihren operativen Ansatz weit überlegen. Denn für sie bilden die durch Selbstreferenz entstehenden Paradoxien nicht die Schlußpointe, sondern den Aus gangspunkt evolutionärer Fortentwicklungen. Das verleiht ihr unter den neueren postmetaphysischen Konstruktionen eine relativ große , Welthaltigkeit".

Dieses Prädikat eignet Luhmanns Texten allerdings weniger dort, wo sie durch die eigene Methodik gedeckt sind, sondern auch und gerade insofern sie dem systematischen Aufriß sozusagen entwischen und eine zweite, vom Standpunkt der Methode aus eigentlich inexistente Materialschicht freilegen. Es gilt mit anderen Worten für die rhetorische Anlage der Systemtheorie, die es ihr erlaubt, wechselweise zwei zugleich voneinander abhängige und miteinander unvereinbare Perspektiven einzunehmen.

Um das zu erläutern, muß man noch einmal etwas weiter ausholen. Autopoiesis kommt bei Luhmann in zwei unterschiedlichen Bestimmungen vor: erstens als Ergebnis von sozialer Evolution - durch funktionale Differenzierung; zweitens aber als Voraussetzung desselben Prozesses. Voraussetzung, insofern Autopoiesis einer simplen Alternative gehorcht: entweder sie findet statt oder nicht. Sie hat keinen Anfang: „Die Autopoiesis des Systems setzt voraus, daß sie immer schon in Gang ist“, sagt Luhmann mit bemerkenswertem Sinn für paradoxe Bündigkeit. ${ }^{21}$ Dieses Paradox verträgt sich aber schlecht mit der evolutionistischen Ausrichtung der Systemtheorie. „Wie soll ein operativ geschlossenes Gesellschaftssystem“, fragt Luhmann selbst, ,das seine eigenen Operationen nicht verwenden kann, um anzufangen oder aufzuhören, sondern entweder autopoietisch operiert oder nicht, evoluieren? Wie soll es allmählich entstehen? Es gibt für Ửbergangslagen kein ,halbes' Leben, kein ,bißchen' Kommunikation. Entweder ein Lebewesen lebt oder nicht. Entweder Kommunikation findet statt oder nicht. Der Begriff verlangt diese kompromißlose Härte." (GdG, munik)

Immer wieder wendet sich der spätere Luhmann gegen die Vorstellung einer „relativen“ und folglich evolutionär steigerbaren „Autonomie“, mithin Umweltunabhängigkeit von Systemen ${ }^{22}$ Er stößt hier im zeitlichen Ablauf auf das gleiche Problem der Undurchlässigkeit der Systemgrenze, von dem bisher mit Blick auf die System/Umwelt-Differenz die Rede war. Der Anfang ist wie das Außen dem System entzogen. Höchstens wird er vom System selbst rückblickend eingesetzt. Allerdings heißt das nicht, daß diese ,Postizipation", wie Luhmann sich ausdrückt $(G d G, 441)$, den Zeitrand und Einsatzpunkt der Autopoiesis des Systems tatsächlich zutreffend rekonstruiert. Im Gegenteil, Luhmann bietet Beispiele dafür, daß es sich bei solchen rückwirkenden Operationen durchweg um legitimatorische Fehldeutungen der Vergangenheit handelt $(G d G, 443 \mathrm{ff})$

21 Niklas Luhmann, Wissenschaft der Gesellschaft (Anm. 15), 595.

22 Vgl. ebd., 290f. „Ich habe frïher selbst diesen Begriff gebraucht und mußte das aufgeben.“ (290)
Der Moment der Instituierung eines operativ geschlossenen Systems ist zugleich die Blindstelle in dem Selbstverhältnis des Systems und in seinen Selbstbeschreibungen. Vom System her läßt sich der Anfang nur denken, insofern er über sich selbst hinaus in eine fiktive Vorzeit verlängert wird - insofern er sich also immer schon ereignet haben soll. Die Vorzeit ist eine Rückprojektion des Systems mit Hilfe seiner bereits instituierten Leitdifferenzen; sie stimmt mit seiner ,technischen' Genese nicht überein. Diese epistemologische Spaltung zieht auch die Position des systemtheoretischen Beobachters in Mitleidenschaft der ja seinerseits nur noch unter den Blickbedingungen der autopoietischen Schließung des Systems beobachten kann. Und genau an dieser Stelle schwenkt Luhmann bezeichnenderweise in das seiner Theorie sonst fremde Register der Erzähltheorie ein: „Die Bestimmung eines Anfangs, eines Ursprungs, einer ,Quelle" und eines (oder keines) ,Davor" ist ein im System selbst gefertigter Mythos - oder die Erzählung eines anderen Beobachters." (GdG, 441, Hervorhebungen A.K.)

Wo es um den Zeitrand der Autopoiesis geht, nimmt die Systemtheorie poetologische Kategorien zu Hilfe. Während das System seinen eigenen Anfang, dessen es nur , von innen habhaft werden kann, mythisch verfehlt, schreibt Luhmann das Erzählenkönnen von Systemanfängen einem ,anderen Beobachter" zu. Dabei hat er selbst sich doch durch seine theoretischen Prämissen grundsätzlich außer Stand gesetzt, dieser andere Beobachter zu sein. Autopoiesis kommt nicht per Gradation - Abschwächung von Fremdkausalität, Zunahme von Eigenkausalität - in die Welt, daran gibt es für Luhmann weder aus der Innennoch aus der Außenperspektive logisch etwas zu rütteln. Sie ist kein Ereigniis ìn einem linearen und objektiven zeitlichen Kontinuum. Sie läßt sich nur denken, wenn man eine Art Zeitfaltung denkt. ${ }^{23}$ Wenn Systeme per Definition autonom sind, müssen sie sich auch selbsttätig instituieren. Sie müssen sich selbst voraufgehen, autonom sein, bevor sie autonom werden. Autonomie, das wußte schon die klassische Bewußtseinsphilosophie, läßt sich nicht durch Heteronomie hervortreiben, und Freiheit kann nur ganz oder gar nicht bestehen. Das heißt nichts anderes, als das Prinzip der Autopoiesis, und zwar gegen alle evolutionäre Stetigkeit, auf sich selbst anzuwenden.

Andererseits betätigt sich Luhmann pausenlos und in immer neuen kulturgeschichtlichen Querschnitten als ein solcher Erzähler. Und in der Suggestion seiner Erzählungen erscheint es ihm als vollkommen evident, die sozusagen perfekte Systematizität von Systemen ans Ende eines langwierigen Prozesses der Perfektibilisierung zu setzen. Dann ist die Rede vom kontinuierlichen Abbau von System-Umwelt-Entsprechungen oder -Repräsentanzen, von „Abweichungsverstärkung“ und einer „mit der Ausdifferenzierung des Systems steigende[n] Auflöse- und Rekombinationsfähigkeit mit entsprechender Abstraktion von Gegenständen $^{\text {‘24 }}$, vom Verzicht auf grenzüberschreitende Ähnlichkeit und von der graduellen Zunahme der System/Umwelt-Differenz. ${ }^{25}$

Semantisch stellt sich das als Auflösung der alteuropäischen Ontologie in Richtung auf plurale, systemrelative Beobachtungsoperationen oder als Übergang von mimetischen zu konstruktiv-kombinatorischen Bezeichnungsweisen wie im Fall des Kunstsystems dar. „Jede Teil-für-Teil-Entsprechung zwischen System und Umwelt (wie man sie zum Beispiel

23 „Unsere Analysen zeigen, daß das Problem des allmählichen Anfangens nur gelöst werden kann, wenn man den zu Grunde liegenden Zeitbegriff revidiert", schreibt Luhmann selbst (GdG, 445), ohne allerdings in dieser Richtung sehr viel weiter zu gehen.

24 Niklas Luhmann, Wissenschaft der Gesellschaft (Anm. 15), 313.

25 Ebd., $473 \mathrm{f}$ 
an Tribalgesellschaften mit Totem-Symbolik beobachten kann) ist unterbrochen. Das Kunstsystem koppelt sich ab. ${ }^{26}$ An anderer Stelle schreibt Luhmann: „Der Schwerpunkt hat sich mit dem Autonomwerden des Kunstsystems von Fremdreferenz auf Selbstreferenz verlagert “27, und wendet so ganz unbefangen einen graduierenden Ausdruck („Autonom werden $^{\prime \prime}$ ) auf den Autonomiebegriff an. Die Grenze, das legen jedenfalls derartige Beschreibungen nahe, war erst unscharf und ist dann, im Verlauf zunehmender funktionaler Ausdifferenzierung, schärfer und schärfer geworden. Manchmal klingt, herabgestimmt durch Luhmanns Habitus funktionalistischer Unterkühlung, fast so etwas wie eine tragische Anthropologie der Moderne durch:

„Stratifizierte Gesellschaften rühmen ihre spezifisch menschliche Ordnung, grenzen sich gegen die Welt der Tiere und der Primitivmenschen $a b$, legen der Unterscheidung aber noch ein religiös-kosmologisch begründetes Sinnkontinuum zu Grunde. Darauf muß die funktional differenzierte Gesellschaft der Moderne dann auch noch verzichten, und die Konsequenz ist, daß sie sich weder mit Regionen noch mit den konkreten, körperlich-mental existierenden Menschen mehr identifizieren kann. Ein Maximum an interner Ungleichheit und Autonomie der Teilsysteme bedingt zugleich ein Maximum an Verschiedenheit von Gesellschaft und Umwelt. Überzeugen kann jetzt nur noch eine scharfe und operativ unüberschreitbare Grenze zwischen System und Umwelt.“ ( $G d G, 617)$

6.

Luhmanns Theorie oszilliert zwischen zwei Logiken, ohne diese Doppelkonditionierung unter Kontrolle $\mathrm{zu}$ bringen. Aus guten und auch empirisch nachvollziehbaren Grïnden nimmt er an, daß die Funktionalität komplexer Systeme sich ihrer operativen Schließung verdankt. Damit wird die Grenze des Systems zu einem Bestandteil seiner eigenen Operationen, und das heißt, daß sie nicht mehr transitorisch gedacht werden kann: weder in synchroner Richtung in Form einer unmittelbaren Wechselkausalität zwischen Umwelt und System noch in diachroner, entwicklungsgeschichtlicher Perspektive. Das ist die eine Vorgabe. Die andere besteht im prominentesten und das Fach überhaupt konstituierenden Begriff der Soziologie, nämlich im Begriff der Differenzierung. Differenzierung umschreib einen zeitabhängigen und von einem wie auch immer genauer bestimmten ,Weniger ${ }^{6} \mathrm{zu}$ einem ,Mehr' hinführenden Prozeß. Mit dem Parameter der Differenzierung wird die Systemtheorie zur Evolutionstheorie. Das Prinzip der Evolution muß aber dann - und hier entstehen extrem heikle logische Probleme - auch auf die Entstehung der Systeme selbs und damit den Prozeß ihrer operativen Abschließung voneinander und von ihrer Umwelt anwendbar sein.

Die Evolution legt ein Kontinuum aus, in das sich dann die Diskontinuitäten räumlicher und zeitlicher Systemgrenzen einzeichnen. Ist aber dieser Schnitt einmal erfolgt, läßt sich rückwirkend das Kontinuum nicht mehr denken. Es wird durch die konstitutive Selbstreferenz, und das heißt externe Voraussetzungslosigkeit, der Systeminnenwelt annulliert. Das System , verschluckt‘ gewissermaßen, kraft seiner Rekursivität, den Fremdkörper seines

26 Niklas Luhmann, Kunst der Gesellschaft (Anm. 19), 372

27 Ebd., 240.
Angefangenhabens. ${ }^{28}$ Jedenfalls hat dieser Fremdkörper keine Systemstelle und nimmt den prekären Charakter eines (gleichwohl unentbehrlichen) Mythos, einer epischen Konstruktion an. Und deshalb steht bei Luhmann im Schatten des rigiden Systematikers stets ein Erzähler, der , trotzdem' erzählt.

Man kann das auch anders beschreiben, nämlich als Schmuggel über die eigentlich unpassierbar gewordenen Souveränitätsgrenzen des Systems. Luhmann befindet sich hier übrigens, das sei nur am Rande vermerkt, in bester abendländischer Gesellschaft. Denn keiner der philosophischen Gründungsakte, an denen die Neuzeit reich ist, von Descartes' Cogito über Kants Transzendentalismus bis hin zur Hegelschen Subjektphilosophie und darüber hinaus, kommt ohne eine klandestine Kehrseite aus, auf der sozusagen althergebrachtes Weltmaterial über die Zäsur, den Abriß, die Nullinie der Selbstsetzung hinübergetragen wird. Und so auch nicht die Systemtheorie.

Unter ihrer oft fugenlos scheinenden Textoberfläche ist Luhmanns Theorie voll von solchen ,illegalen' Grenztransfers. ${ }^{29}$ Sie greift überdies in verstärktem Maß auf theoretische Kategorien zurück, die durch ihre grenzüberschreitende Operationsfähigkeit gegenstrebig zur Schließung der Grenze agieren. Das betrifft vor allem den Begriff der „strukturellen Kopplung“, der auf der Wegstrecke von den Sozialen Systemen zur Gesellschaft der Gesellschaft immer wichtiger wird und ein Großteil der Integrationsprobleme zu kompensieren hat, die sich aus der methodischen Verschärfung der System/Umwelt-Differenz ergeben. ${ }^{30}$

Das kann an dieser Stelle nicht näher erörtert werden. Es soll genügen, sich auf den Schmuggel ' in diachroner, evolutionärer Hinsicht zu beschränken. Laut Definition sind Systeme selbstreferentiell und produzieren Fremdreferenz durch Selbstreferenz. Laut ,Erzählung ' aber entsteht Selbstreferentialität unter Abbau von Fremdreferenz: Autopoiesis durchschneidet und versperrt (zuvor bestehende) System/Umwelt-Bezüge. Als Ergebnis von Differenzierung geht sie aus einem System/Umwelt-Verhältnis hervor, das - man kann es wenden, wie man will ${ }^{31}$ - einfacher, transitiver, analoger, synchroner oder mit einem Wort enger gewesen sein muß.

28 Systematische Überlegungen dazu in meiner Arbeit Körperströme und Zeichenverkehr. Mediologie des 18. Jahrhunderts, München 1999 (im Erscheinen).

29 Dazu nur ein Beispiel: der Begriff einer positiven Realität, den die Kunst der Gesellschaft als Kontrastfolie mitführt. Auch im Bereich der Kunst entsteht, Luhmann zufolge, „Ordnung auf der Basis einer Selbstirritation; aber das ist nur möglich, wenn vorab durch Ausdifferenzierung eines Mediums für Kunst entschieden ist, daß es dabei nicht nur um das geht, was sich als Wirklichkeit ohnehin [!] zeigt. Kunst entschieden ist, daß es dabei nicht nur um das geht, was sich als Wirklichkeit
Die wirkliche Welt ist immer so, wie sie ist, und nie anders." (ebd. (Anm. 19), 237)

$30 \mathrm{Vgl}$. $G d G, 776 \mathrm{ff}$. Es handelt sich dabei sozusagen um Rückkopplungsscharniere - etwa die Staatsverfassung als Bindeglied zwischen politischem und Rechtssystem. Strukturelle Kopplung übernimmt allerdings nur die Koordination zwischen Systemen (genauer gesagt: sie legt Bandbreiten ihrer wechselseitigen Irritierbarkeit fest), nicht zwischen Gesellschaft und Umwelt überhaupt. Sie dient dazu, die Integration der Funktionssysteme vorab zu garantieren und nicht von evolutionären Anpassungen im Sinn Darwins abhängig zu machen, die durch Luhmanns radikale Fassung des Begriffs der Systemautonomie unmöglich geworden sind ( $G d G, 446)$.

Im übrigen tauchen rings um diesen Terminus alle oben diskutierten Probleme des Verhältnisses zwischen Evolution und Autopoiesis wieder auf. Strukturelle Kopplungen sollen einen von Luhmann angenommenen vorfunktionalen Gleichlauf der Teilsysteme auflösen und analoge in digitole Verhältnisse umformen" (GdG,101). Sie gehorchen da zugleich beschreibt und dementiert.

31 Znd dics trotz der Ǘ denten.

r Uberlegungen, die Luhmann selbst in der Gesellschaft der Gesellschaft anstellt $(446 f, 615 f)$. 
Interessanterweise gerät so der Konstruktionszwang der Theorie in das Fahrwasser all der sentimentalischen Selbstbeschreibungen, die von Anbeginn an zur Moderne gehören. Was Luhmann technisch als Auflösung von Punkt-für-Punkt-Entsprechungen zwischen System und Umwelt bezeichnet, braucht nur geringfügig umformuliert zu werden, um darin jenen Verlust von Nähe und Erfahrungsunmittelbarkeit wiederzuerkennen, über den moderne Autoren klagen, seitdem sie sich über die Irreversibilität des Geschichtsverlaufs Rechenschaft geben.

Selbst in dem spröden und weitgehend funktionalitätskonformen Duktus der Systemtheorie ist die Häufigkeit derartiger Nicht-mehr-Konstruktionen auffällig. Sie stellen sich geradezu zwangsläufig her, gegen die dem Text aufgegebene Intention. Es scheint, als ob evolutionistische Entwürfe eine fast grammatikalische Tendenz dazu hätten, in einer Geschichtsphilosophie der Abkühlung, des Zuwachses von Ferne, Indirektheit oder Beziehungskomplikation einzumünden

Das hat nichts mit empirischen Verfallskurven zu tun, auch nichts mit den Evidenzen einer systemtheoretischen Beobachterposition. (Wie und von welchem Ort der Beobachtung aus sollten alteuropäische und angeblich geringer ausdifferenzierte Wissensbestände referenzstärker, umweltnäher gewesen sein als diejenigen der modernen Funktionssysteme?) Es erklärt sich vielmehr aus Zwängen der Narration. Wer Evolutionsgeschichten erzählt, kann vermutlich nicht anders erzählen. Dem Vergangenen kommt seine direktere Referentialität nicht aus substantiellen, sondern erzähltechnischen Gründen zu. In evolutionären Wissenssystemen ist Referenz offenbar nur als Ausgangspunkt der das System konstituierenden Prozedur, als ein durch seine Einholung in die Welt der Zeichen fortschreitend distanziertes Relikt beschreibbar. Semiosen, Zeichenketten, lassen das Bezeichnete retrospektiv, als ein ihnen vorausliegendes Wesen, erscheinen. Der Referent ist dann das, woran sich das Wissen erinnert. Und wenn es von seinen Erinnerungen erzählt, gestaltet es die operative Zeitdifferenz, durch die es sich selbst generiert, zu einem Mythos vom Schwinden der Referentialität um.

Wahrscheinlich bietet die Systemtheorie ein besseres Instrumentarium als die mit ihr konkurrierenden Theorien, um die logischen Implikationen solcher Probleme der, Systemsemantik' durchzuspielen. Andererseits ist die Systemtheorie selbst ein semantisches Unternehmen und in dieser Eigenschaft in die gleichen Probleme verstrickt. Sie wird ihre Funktionslogik um eine kulturelle Nachbardisziplin erweitern müssen: die Narratologie. 